Tropical Journal of Pharmaceutical Research March 2014; 13 (3): 459-467

ISSN: $1596-5996$ (print); 1596-9827 (electronic)

(C) Pharmacotherapy Group, Faculty of Pharmacy, University of Benin, Benin City, 300001 Nigeria.

All rights reserved.

Available online at http://www.tjpr.org

Review Article

http://dx.doi.org/10.4314/tjpr.v13i3.22

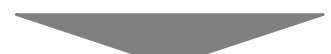

\title{
Nasal Inserts for Drug Delivery: An Overview
}

\author{
Evren Algin-Yapar \\ The Ministry of Health of Turkey, Turkish Medicines and Medical Devices Agency, Sögütözü Mahallesi 2176. Sokak No:5, \\ 06520 Çankaya Ankara, Turkey.
}

*For correspondence: Email: evren.yapar@yahoo.com, algin@pharmacy.ankara.edu.tr_Tel: +90. 532.38256 86; Fax: +90. 312. 2183585

Received: 18 December 2013

Revised accepted: 19 February 2014

\begin{abstract}
Nasal mucosa, owing to its high vascularity and thin epithelial membrane, has been widely explored for drug administration in the last two decades. Within that period various nasal dosage forms have been researched and developed. Among them, nasal inserts are easily prepared by lyophilization or gelsification, allow easy dosing with a high potential for systemic administration, enable avoidance of hepatic first pass metabolism, and make possible the release of active ingredient in a controlled manner. In this review, the benefits, limitations and absorption mechanisms of the nasal route, as well as findings from nasal insert-related studies are addressed.
\end{abstract}

Keywords: Nasal drug delivery, Insert, Mucoadhesion, Absorption, Lyophilization, Gelsification.

Tropical Journal of Pharmaceutical Research is indexed by Science Citation Index (SciSearch), Scopus, International Pharmaceutical Abstract, Chemical Abstracts, Embase, Index Copernicus, EBSCO, African Index Medicus, JournalSeek, Journal Citation Reports/Science Edition, Directory of Open Access Journals (DOAJ), African Journal Online, Bioline International, Open-J-Gate and Pharmacy Abstracts

\section{INTRODUCTION}

The nasal mucosa, due to its large surface area, porous endothelial membrane, and high vascularization, has been considered as a potential administration route to achieve faster and higher level of drug absorption with the avoidance of first-pass metabolism and ready accessibility. Nasal administration of active molecules including peptide and proteins for systemic medication and immunization has been widely investigated in recent years. Benefits, limitations and absorption mechanism of nasal route from different points of view have also been evaluated by investigators [1-4]. Bioadhesive nasal drug delivery systems have been mostly designed as powders, micro- and nanoparticulate forms, hydrogels and inserts. This review on nasal inserts is focused on nasal absorption, its benefits, limitations and mechanism of drug delivery.

\section{NASAL DRUG DELIVERY}

\section{Benefits of nasal delivery}

The benefits of nasal delivery [1] indicate that it is a good alternative to parenteral route, with its easy of administration (needle-free application). Thus, there is no need for trained personnel which means self-medication is possible; in addition, it does not contribute to biohazardous waste since special containers are not required $[4,5]$. With regard to absorption, good penetration of lipophilic molecules and low molecular weight $(\mathrm{Mw})$ drugs, rapid and fast onset of action due to relatively large absorption surface area and high vascularization are accepted as important advantages [6]. Protection of drugs from the conditions of the gastrointestinal tract that may cause chemical and enzymatic degradation and protection from hepatic first pass metabolism results in dose reduction compared to oral delivery [1]. The direct delivery potential of drugs 
to the central nervous system via the olfactory region which allows by-passing the blood brain barrier is another important advantage of nasal route [7]. Thus, it can be important for drugs that are used for crisis management such for pain and centrally acting drugs where the direct pathway from the nose to the brain might provide a faster and more specific therapeutic effect [8]. Another advantage of this route is the direct delivery of vaccine to lymphatic tissue and induction of a secretory immune response at distant mucosal site [9]. Nasal route may also be an alternative for drug molecules unpalatable taste in the case of pediatric drug delivery.

\section{Limitations of nasal delivery}

Reasons for limitations of nasal route can be summarized as low bioavailability, mucociliary clearance, enzymatic degradation and nasal pathophysiology [1]. Nasal absorption can be affected by the physicochemical characteristics of the administered drug such as Mw, solubility, dissolution rate, partition coefficient, charge, pKa, particle size and polymorphism [10]. The physicochemical properties of a drug can be altered by the presence of some enhancers in the formulation by modifying drug solubility, partition coefficient, or ionic interactions with the drug [4]. Generally, polar and low Mw drugs show low bioavailability of about 10 and $1 \%$, respectively [8]. The most important factor limiting nasal absorption of polar and large $\mathrm{Mw}$ drugs is low membrane permeability [1] and this can be overcome by incorporating absorption enhancers in the formulation [2].

In this context, absorption enhancers can be classified as chemical and physical enhancers; while the former act by disrupting the nasal mucosa irreversibly, the latter acts by forming a gel to effect nasal clearance reversibly. Chelating agents, bile acid salts, fatty acids, surfactants and preservatives can be classified as chemical enhancers whereas gelation agents are physical enhancers. In some cases, osmolarity and $\mathrm{pH}$ may also have enhancing effect.

Enhancing mechanisms include decrease in mucociliary clearance, reduction of mucus viscosity or elasticity, inhibition of enzyme activity, opening of tight junctions, and solubilization or stabilization of the drug molecule [11-24]. Mucociliary clearance can be defined as combined actions of the mucus layer and the cilia, which is an important factor in the physiological defence of the respiratory tract against inhaled hazardous particles [13]. Nasal mucociliary clearance can be stimulated or inhibited by active ingredients, excipients, absorption enhancers or preservatives, which facilitate the delivery of the drug to the absorption site [14]. Fast clearance of formulation results in low absorption of drug for liquid and powder formulations that are not bioadhesive. Low halflife for clearance is another factor for low membrane transport $[15,16]$. The use of bioadhesive excipients in the formulations is an approach to overcome rapid mucociliary clearance. Depositing the formulation in the anterior, less ciliated part of the nasal cavity may also reduce the clearance and thus improved the absorption [17,18]. Metabolic enzymes in the nasal mucosa may also limit the bioavailability of some drugs particularly peptides and proteins [19]. The possibility of enzymatic degradation in the lumen of the nasal cavity or during passage through the epithelial barrier containing exopeptidases and endopeptidases is another but less considered factor for low bioavailability of peptides and proteins [20]. The use of enzyme inhibitors and/or saturation of enzymes may be possible approaches to overcome this barrier [21]. The limitations include the risk of local side effects and irreversible damage of the cilia on the nasal mucosa which may be related to the drug or the other substances in dosage forms can be examplified as disruption or even dissolution of the nasal membrane by using absorption enhancers in high concentrations. Nasal atrophic rhinitis and severe vasomotor rhinitis can reduce and furthermore nasal polyps and cancers may alter the absorption from the nasal cavity in different ways [3].

\section{Mechanism of nasal drug absorption}

The mechanism of nasal drug absorption is affected by several factors [1]. For systemic drug delivery, the anatomically most important region in the nose is the respiratory region between the three distinct functional regions identified as vestibular, respiratory, and olfactory [3]. Mucus is the first barrier in the absorption of drug from the nasal cavity [16]. Anatomical cross-section of the nasal region and mucosa sections of nasorespiratory region is illustrated in Figure 1. While small, unchanged particles easily pass through this layer, the large or charged particles probably hardly cross it. Mucin, the principal protein in mucus, has the potential to bind to solutes and hampering diffusion. However, structural changes in the mucus layer can be formed by environmental changes (i.e., $\mathrm{pH}$, temperature, etc). Next, drug absorption through the mucosa includes transcellular or simple diffusion across the membrane, and paracellular transport via movement between cell and transcytosis by vesicle carriers [16]. 


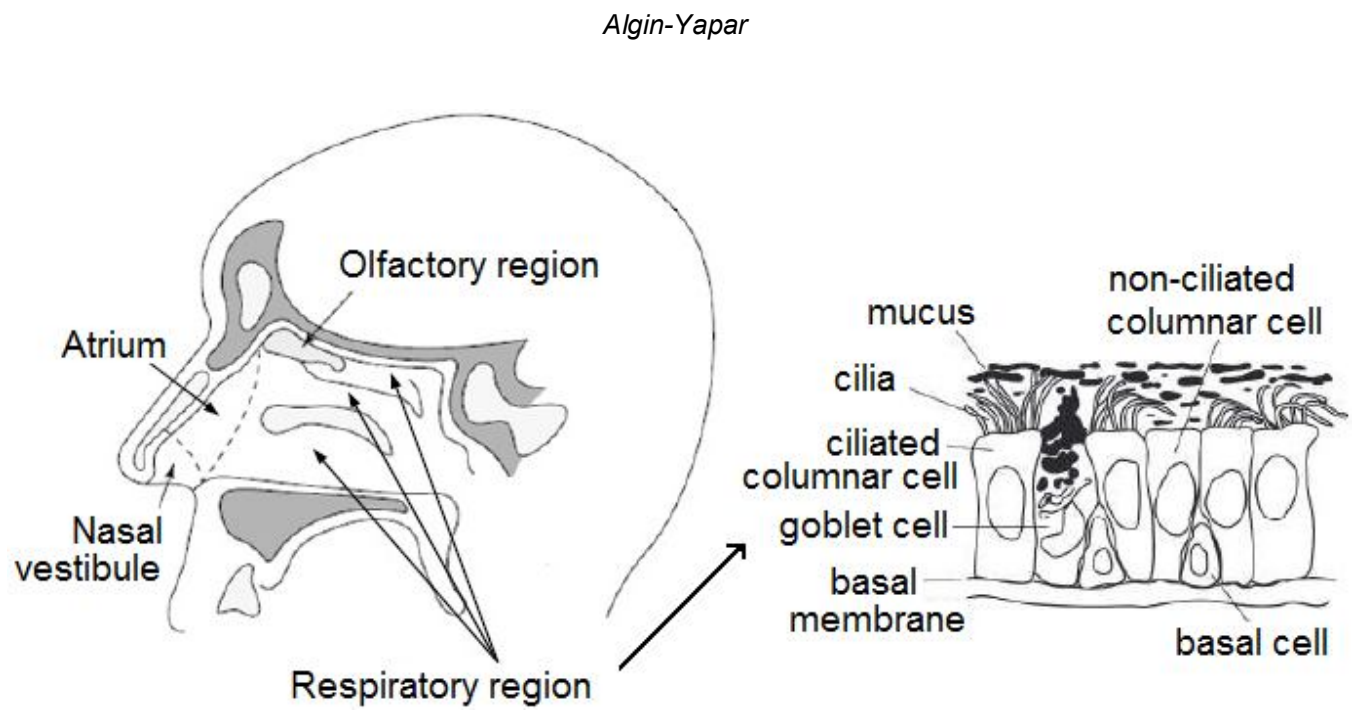

Figure 1: Anatomical cross-section of nasal region and mucosal subsection of naso-respiratory region (modified, courtesy of ref 24)

Potential metabolisms before reaching the systemic circulation and limited residence time in the cavity are main challenges for drug absorption. Although several mechanisms have been proposed, the paracellular route and transcellular route have been considered predominantly [1]. Briefly, paracellular route involves aqueous route of transport, which is slow, passive and characterized with an inverse log-log correlation between absorption and Mw of water-soluble molecules particularly poor bioavailability for Mw greater than 1000 Daltons. Transcellular route involves transport through a lipoidal route which is responsible for the transport of lipophilic molecules dependent on their lipophilicity. Molecules cross cell membranes by an active transport route via carrier-mediated means or transport through the opening of tight junctions as well [22].

Physicochemical properties such as ionization, lipophilicity, etc., surface charge and hydrophobicity of molecules are important factor besides the molecular size [23]. In the case of small and large hydrophilic drugs may be poorly permeable across nasal epithelium and may show an insufficient bioavailability. It is possible to greatly improve their absorption if they are administered in combination with absorption enhancers which induce reversible modifications on the structure of epithelial barrier [24,25]. The low residence time of the drug in the nasal cavity is a limitation of this route, which may affect absorption and bioavailability; thus, this requires modification to provide longer residence time by improving the bioadhesion properties of the formulation [26,27]. Increasing the viscosity of the formulation or using microparticulate systems may also prolong residence time in the nasal cavity [28].
Briefly, properties desired for nasal bioadhesive formulations can be summarized as, (a) good adherence to nasal mucous membrane, and ability to absorb mucus; (b) form a viscous layer or show a slow clearance; and (c) protect active agent/drug or release it slowly $[3,29,30]$.

\section{NASAL INSERTS}

Nasal bioadhesive dosage forms can be classified as bioadhesive powders [31,32], micro and nanoparticulate systems [33], hydrogels [3436] and inserts [37-60]. Among nasal inserts, bioadhesive solid dosage forms are promising for prolonged drug delivery that allow easy dosing with high potential for systemic administration. The principle of nasal insert can be explained as absorption of nasal fluid from the mucosa following its application and forms a gel which adheres to the nasal mucosa owing to its bioadhesive property and thus acts as release controlling matrix providing a sustained drug delivery. Since the gel dissolves and/or move towards the nasopharynx there is no need to remove insert mechanically after it is depleted of drug [3,26,37-39]. Nasal inserts can be prepared by either Iyophilization or gelsification [26,40-43]. Gelsification can briefly identify that the method either electrostatic complexation or ionotropic gelation in which harsh cross-linking agents is used in emulsion cross-linking [40,41]. However, lyophilization is more usual technique for the preparation of nasal inserts and it is one of the applied methods for drying of solids either in the form of aqueous solution or rarely in the form of aqueous suspension by using freeze dryers $[42,43]$. The process of lyophilization involves a container closed with an impermeable membrane pierced with one or more holes through which the material in the container can be lyophilized. Holes are large enough to allow the escape of water vapor from the material that is kept in the container. Resultant insert consist of a drug 
embedded sponge like hydrophilic polymer matrix $[26,37,38]$ which release the drug mainly following the steps; at first inserts come into contact with the high vascularised nasal mucosa, then the polymer in its structure absorb the mucin, quickly transforms gel and thus provide a sustained an emissions and/or controlled release profile [38,54]. A lyophilized nasal insert in a bullet shaped with internal diameter of $1 \mathrm{~cm}$ at the tube mouth and $3.5 \mathrm{~cm}$ in length is presented in Figure 2.

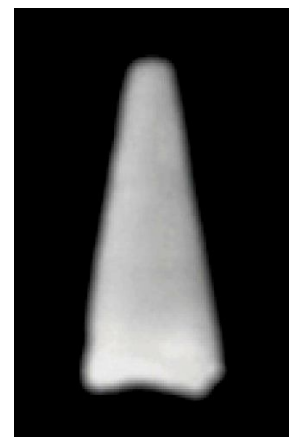

Figure 2: Shape of a freeze-dried nasal insert [54]

This novel technique is a convenient way of lyophilizing nonsterile products in their primary packaging and increases the potential for the development of lyophilized formulations for nonparenteral applications [44].

\section{Overview of Works on Nasal Inserts}

In case of dosage form development for nasal delivery, properties of drug, delivery system and nasal physiology should be considered from the beginning. In manufacturing approaches freezedrying method has usually been applied for the manufacture of unit dose, fast dissolving dosage forms and nasal inserts [45]. In the context of the foregoing, nasal delivery studies are few despite its many advantages, probably owing to some limitations. However, studies on nasal inserts of some active molecules and proteins using mucoadhesive polymers such as cellulose derivatives, acrylic acid derivatives, polysaccarides and gums are outlined here [38,46-62].

A short-acting $\beta 2$-adrenergic receptor agonist, salbutamol sulfate, has been prepared as nasal inserts using hydroxypropyl methylcellulose (HPMC), carboxymethylcellulose sodium, sodium alginate and chitosan as gel-forming polymers in order to investigate their physicochemical properties. The interaction observed by differential scanning calorimetry between drug and oppositely charged polymers (carboxymethylcellulose sodium, sodium alginate) resulted in zero order release of the drug for $>10 \mathrm{~h}$. However inserts prepared with all the polymers gave acceptable mucoadhesive and extended release properties which could be useful for nasal delivery of salbutamol sulfate [46].

A nasal insert formulation prepared by lyophilisation of a viscous HPMC gel solution designed by Mclnnes et al [47] to overcome rapid mucociliary clearance problem.

vitro prolonged nicotine release from the lyophilised nasal inserts compared to powder and liquid spray formulations was investigated and then in vivo comparison were carried out on sheeps in a randomised four-way cross-over study. Though high variability in the pharmacokinetic data, promising prolonged plasma profiles which need to be improved were obtained with inserts [47]. A follow-up study focused on a larger molecule, insulin, administration to healthy male volunteers in order to quantify nasal residence of conventional nasal spray solution and lyophilised nasal insert formulations (1 - $3 \%$ HPMC) using gamma scintigraphy [48]. While conventional nasal spray deposited in the posterior nasal cavity with a rapid clearance half-life of $9.2 \mathrm{~min}$, nasal inserts ( $2 \%$ HPMC) extended nasal residence time of 4 - $5 \mathrm{~h}$ with rapid adhesion and without overhydration [48].

The influence of the physicochemical properties of drug, drug loading and composition of the release medium on drug release from in situ gelling nasal inserts was investigated by Bertram and Bodmeir [38]. Carrageenan and HPMC $\mathrm{K} 15 \mathrm{M}$ containing sponge-like nasal inserts with the model drugs oxymetazoline $\mathrm{HCl}$, diprophyllin, and acetaminophen were prepared by lyophilization. Drug release from nasal inserts was governed by drug solubility, physical state of drug in the polymer matrix, and interactions between drug and polymer. Additionally oppositely charged polymers and drugs were influenced by electrostatic drug-polymer interactions and by the composition of the release medium [38].

Investigation of in situ gelling inserts based on bioadhesive hydrophilic polymers [carrageenan, Carbopol, chitosan, HPMC K15M and E5, sodium alginate, sodium carboxy methylcellulose (NaCMC), polyvinyl pyrrolidone (PVP) 90, xanthan gum] including oxymetazoline $\mathrm{HCl}$ showed that sponge-like structure was obtained with amorphous polymers and the hardness of inserts was increased with the glass transition temperature of the polymers [49]. Bioadhesion potential was governed by the polymer's ability to interact with mucin and inserts prepared from low Mw polymers which also resulted in polymer 
dissolution and fast drug release. Drug release from high Mw polymers was a complex interplay of water uptake, osmotic forces, and electrostatic interactions between drug and polymer. Different drug loadings of carrageenan inserts resulted initially in similar (up to $3 \mathrm{~h}$ ) and then in a decreased drug release with increasing drug loading. The decrease in freely water-soluble oxymetazoline $\mathrm{HCl}$ supports the statement on electrostatic interactions between polymer and drug. The slower release from the inserts at the higher drug loading was attributed to possibility of slower water uptake due to complex formation [49].

Work on the effect of polymer Mw and polymer blends on the release of oxymetazoline $\mathrm{HCl}$ and diprophyllin from in situ gelling nasal inserts showed that sonication time did not significantly afftect drug release from carrageenan inserts [50]. Release of drugs was slow from high $\mathrm{Mw}$ and increasing sodium alginate content of inserts. The bioadhesion potential of sodium alginate inserts was strongly reduced in the case of low Mw. The use of polymer blends to control the drug release from nasal inserts was superior to the use of polymers of different Mw [50].

A 32 factorial design was used for tramadol $\mathrm{HCl}$ nasal inserts to investigate the combined effect of two independent formulation variables in the preparation of inserts. The Carbopol 971P: polycarbophil ratio (X1) and the amount of polyethylene glycol (PEG) 400 (X2) were selected as independent variables. Batches were evaluated for water uptake, mass loss, thickness, bioadhesion potential and drug diffusion across the nasal mucosa. Formulation variables were found effective on the thickness and water uptake of the nasal inserts and changed in variables resulted as a mass loss response positively. The bioadhesion potential was significantly dependent on the Carbopol 971P: polycarbophil weight ratio. Diffusion across the nasal mucosa showed a matrix-type profile and the $T 50 \%$ (Time required to release $50 \%$ of the drug from inserts) was found to increase as the concentration of polycarbophil increased. This systematic approach was found to be useful in investigating the effect of the formulation processing variables for formulation development [51].

Nasal insert of verapamil $\mathrm{HCl}$ having high first pass metabolism was aimed to improve the bioavailability and prolonged release of drug. Inserts prepared by sodium alginate at a concentration of $4.5 \% \mathrm{w} / \mathrm{w}$ gave the best Higuchi kinetic model that fitted prolonged release of drug. Ex vivo studies show that PEG 400 which was used as penetration enhancer in inserts increased the release of drug significantly [52].

Werner et al [53] was studied to incorporate estradiol complexed with methyl- $\beta$-cyclodextrin $(M \beta C D)$ into in situ gelling nasal inserts. It was found that the addition of $M \beta C D$ to carrageenan solutions significantly increased the viscosity that could be attributed to the dehydration of carrageenan, cations impurities in $M \beta C D$, and interactions. In vitro drug release was independent of estradiol dose or complex molar ratio. Administration of inserts to male rats showed that nasal inserts stayed for $6 \mathrm{~h}$ after administration and led to a more gradual absorption of estradiol with lower peak serum levels compared to solution and microparticle [53].

Combination of xanthan gum and guar gum was used to prepare the nasal inserts and the effect of blend ratio of xanthan gum and guar gum on drug release from in situ gelling nasal inserts and on insert properties was studied by Dehghan and Girase [54]. The viscosities of xanthan gum in combination with guar gum were observed to be higher than that of single polymer solutions. This is due the synergistic rheological interaction between xanthan and guar gum which reached to maximum both in synergy and viscosity in case of 1:1 xanthan: guar gum ratio. The best nasal inserts formulation containing xanthan gum and guar gum ratio 1:5, showed good release as well as bioadhesion which may result in an increase in the nasal residence time [54].

Influenza split vaccine including nasal insert was prepared as vaccine delivery system [55]. Due to the high $\mathrm{Mw}$ of the proteins, in vitro release from either inserts or polymer solutions was not successful. The release rate was dependent on the polymer owing to differences in viscosity and possible polymer-protein interactions. While xanthan gum, a negatively charged polymer enhanced the serum IgG and thus the nasal IgA response in in vivo studies the poly-l-arginine and cationic lipid were found as the best performing adjuvants. However, the addition of polymers and the freeze-drying process were not significantly affect the specific activity of the major vaccine protein, hemagglutinin. In vivo studies showed that rat immunization with solid nasal inserts based on xanthan gum containing the influenza vaccine, showed similar IgG levels with the pure liquid formulation [55].

Bacteriophage include nasal delivery was studied by some researhers [56-58]. Puapermpoonsiri et al [56] showed that the stability of the bacteriophage in the nasal inserts was far 
greater than for encapsulated phage in polyestermatrices, wherein no lytic activity was observed after 1 week and Wright et al [57] showed that the bacteriophage titer in the inserts after 12 months may still represent a therapeutic dose. The first controlled clinical trial of a therapeutic bacteriophage preparation showed efficacy and safety in chronic otitis because of chemo-resistant P. Aeruginosa [56]. Following bacteriophage therapy of methicillin-resistant Staphylococcus aureus with a nasal insert was studied by Alfadhel et al [58]. In this study it has shown that lyophilization of bacteriophages in a viscous solution of $1-2 \% \mathrm{w} / \mathrm{v}$ HPMC with/without the addition of $1 \% \mathrm{w} / \mathrm{v}$ mannitol, gives highly porous nasal insert matrix. Fluorescently labeled bacteriophages were observed to be homogenously distributed throughout the wafers of the dried matrix. The results show that such an insert could be useful for the eradication of methicillin-resistant Staphylococcus aureus resident in the nose [58].

Luppi et al [59] studied mucoadhesive nasal inserts based on chitosan/hyaluronate polyelectrolyte complexes prepared at various $\mathrm{pH}$ and at different molar ratios. Results indicated that the selection of complex preparative conditions allows modulation of insert swelling and mucoadhesion ability. Drug release behaviour from inserts was different for vancomycin and insulin. It was suggested that chitosan/hyaluronate polyelectrolyte complexes can be used for the delivery of peptide and protein drugs as nasal inserts [59]. In a subsequent study Luppi et al [60] investigated the development of chitosan/pectin based chlorpromazine hydrochloride nasal inserts to improve bioavailability of antipsychotic drugs. Chitosan/pectin polyelectrolyte complexes showed that higher amount of pectin compared to chitosan resulted as more porous inserts which improved ability of water uptake and capacity of mucoadhesion.

As can be seen in Figure 3, pectin showed greater mucoadhesive capacity compared to chitosan. This was attributed to the different water uptake ability of pectin and chitosan, thus providing a more or less efficient chain mobility and physical entanglement with mucus, respectively by researchers. For the same reason, high percentages of pectin showed the best in vitro mucoadhesion ability which verifies the formation of polyelectrolyte complexes between chitosan and pectin at $\mathrm{pH}$ values in the vicinity of the pKa interval of the two polymers. This work has contributed to the understanding of chitosan/pectin polyelectrolyte complex formation and complexation with chlorpromazine hydrochloride and will be furthered by performing intranasal absorption studies in animal models [60].

Hydration and adhesion parameters, which are important to assess the performance of nasal bioadhesive formulations can be evaluated using in vitro tests designed in an attempt to mimic relevant processes in the nasal cavity. Scanning electron microscopy as an in vitro test give the oppurtunity of evaluation of the lyophilisate internal structure which need to be highly porous to provide the ideal pathway for rehydration. Vapour sorption analysis can be used for substantial weight gain of the lyophilisates with exposure to $95 \%$ relative humidity. Agar was used as a synthetic mucosal model designed to provide a standardised quantity of water available for rehydration of the formulations. Dynamic adhesion and texture analyser sliding

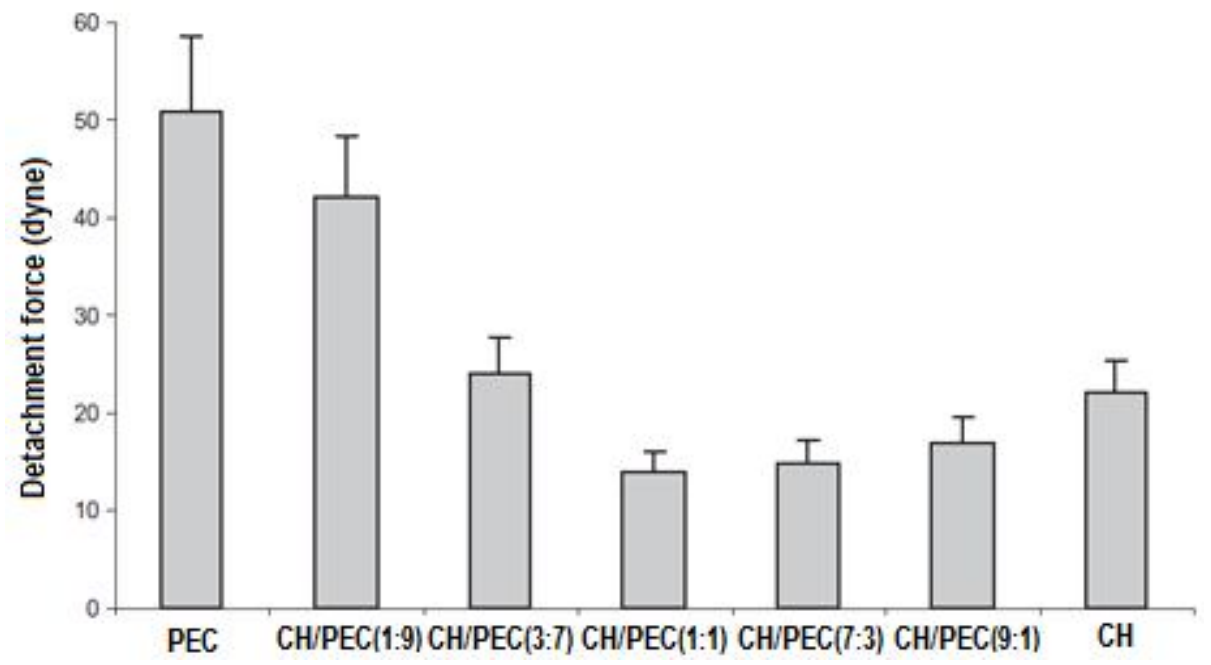

Figure 3: Mucoadhesive capacity, expressed as detachment force (mean $\pm S D, n=$ 3 ) of chitosan hydrochloride, pectin and chitosan/pectin complexes at pH 5.5 [60]. 
tests can be use to quantify different aspects of the spreading and adhesion of the hydrating formulations on the synthetic mucosal surface. Confocal microscopy analysis can be use for the examination of the lyophilised formulations which allow visualisation and quantification of the initial rate of water ingress. The use of a combination of the above methods indicates that would be useful for the development and evaluation of insert formulations by mimicking the dynamic conditions of the nasal cavity [61].

An in vitro method based on LC with fluorescence detection has been developed by Mercolini et al, for the determination of insulin in innovative formulations consisting of microparticles and inserts for oral and nasal drug administration, respectively. Nasal inserts consisted of chitosan/hyaluronate polyelectrolyte complexes which were loaded with insulin. The employment of fluorescence detection provided high selectivity, with no interference from the matrix. Insulin content obtained from a single nasal insert was complying with preparative protocols [62].

The hypothesis of human nasal RPMI 2650 cells grown at an air-liquid interface which is a feasible model for drug transport studies via the nasal route has been tested and evaluated with confocal microscopy by Bai et al [63]. RPMI 2650 cells were cultured in Eagle's minimal essential medium at air-liquid and also liquid-liquid interfaces. The expression of tight junction proteins (differentiation markers) in cells of the different monolayers was studied by Western blot analysis and confocal microscopy. Confocal microscopic studies showed that RPMI 2650 cells grown at an air-liquid interface form polarized monolayers with the cells interconnected by tight junction proteins. This human nasal cell line model indicated that could be a useful tool for in vitro screening of nasal drug candidates [63].

Excised and cultured human or animal tissues for nasal drug absorption and metabolism researches have increased however most reported are based on animal tissues. Agu and Ugwoke [63] presented comparative treatises on various in situ and ex vivo nasal models with their benefits, limitations, applications in preclinical drug development, and in vivo/in vitro correlation [64].

A majority of the products available are used for the treatment of allergic rhinitis, migraine, cold, pain etc. Although various formulations given by the nasal route includes nasal gel, spray, powders etc, there has not been any commercially available nasal insert yet. Not only has the above mentioned indications but also for many others nasal delivery systems are considered a promising alternative to other drug delivery systems [65].

\section{CONCLUSION}

Nasal route is a good alternative to parenteral route for drug administration, and nasal inserts which can be prepared using a simple and reproducible production method. Their bioadhesive character and prolonged release pattern can facilitate commercial production of formulations of active ingredients which otherwise are given paranterally.

\section{REFERENCES}

1. Jadhav KR, Gambhire MN, Shaikh IM, Kadam VJ, Pisal SS. Nasal drug delivery system-Factors affecting and applications.Current Drug Therapy 2007; 2: 27-38.

2. Illum L.Transport of drugs from the nasal cavity to the central nervous system. Eur J Pharm Sci 2000; 11: 1-18.

3. Basu S, Bandyopadhyay AK. Nasal drug delivery: An overview. Int J Pharm Sci Tech 2010; 4(1): 1-20.

4. Deshpande $T$, Masareddy $R$, Patil A. Nasal drug delivery-a review. RGUHS J Pharm Sci 2012; 2(1): $24-37$.

5. Pontiroli AE, Calderara A, Pozza G. Intranasal drug delivery: potential advantages and limitations from a clinical pharmacokinetic perspective. Clin Pharm 1989; 17: 209-307.

6. Striebel HW, Pommerening J, Rieger A. Intranasal fentanyl titration for postoperative pain management in an unselected population. Anaesthesia 1993; 48(9): 753-757.

7. Talegaonkar S, Mishra PR. Intranasal delivery: An approach to bypass the blood brain barrier. Indian J Pharmacol 2004; 36(3):140-147.

8. Illum L. Nasal drug delivery: new developments and strategies. Drug Discov Today 2002; 7(23): 11841189.

9. Davis SS. Nasal vaccines. Adv Drug Deliv Rev 2001; 51: 21-42.

10. Behl CR, Pimplaskar HK, Sileno AP, deMeireles J, Romeo VD. Effects of physicochemical properties and other factors on systemic nasal drug delivery. Adv Drug Del Rev 1998; 29: 89-116.

11. Singh AK, Singh A, Madhav NVS. Nasal cavity, a promising transmucosal platform for drug delivery and research approaches from nasal to brain targetting. J Drug Deliv Therapeutics 2012; 2(3): 22-33.

12. Fisher A, Illum L, Davis S, Schacht E. Di-iodo-L-tyrosine labeled dextrans as molecular size markers of nasal absorption in the rat. J Pharm Pharmacol 1992; 44: 550-554.

13. Marttin E, Schipper NGM, Verhoef JC, Merkus FWHM. Nasal mucociliary clearance as a factor in nasal drug delivery. Adv Drug Del Rev 1998; 29: 13-38.

14. Bermejo M, Gonzalez-Alvarez I. How and where are drugs absorbed? Pharmaceutical Sciences Encyclopedia: Drug Discov Develop Manuf 2010; 1: 1-32.

15. Soane RJ, Frier M, Perkins AC, Jones NS, Davis SS, Illum $L$. Evaluation of the clearance characteristics 
of bioadhesive systems in humans. Int $J$ Pharm 1999; 178: 55-65.

16. Illum L. Bioadhesive formulations for nasal peptide delivery. In: Mathiowitz E, Chickering DE, Lehr CM. [Eds.], Fundamentals, Novel Approaches and Development, Marcel Dekker, New York, pp. 1999, 507-539.

17. Kublik H, Vidgren MT. Nasal delivery systems and their effect on deposition and absorption. Adv Drug Deliv Rev, 1998; 29: 157-177.

18. Harris AS, Nilsson IM, Wagner ZG, Alkner U. Intranasal administration of peptides: nasal deposition, biological response, and absorption of desmopressin. J Pharm Sci 1986; 75(11):10851088.

19. Irwin WJ, Holbrook PA, Dey MJ. The stability of peptides in nasal enzymic systems. Int J Pharm 1995; 113 . 25-37.

20. Lee VHL. Enzymatic barriers to peptide and protein nosebrain pathway for psychotropic peptides: evidence absorption. CRC Crit Rev Ther Drug Carrier Syst 1988; 5: 69-97.

21. Morimoto K, Miyazaki M, Kakemi M. Effects of proteolytic enzyme inhibition on nasal absorption of salmon calcitonin in rats. Int J Pharm 1995; 133: 1-8.

22. Aurora J. Development of nasal delivery systems: $A$ review. Drug Deliv Technol 2002; 2(7): 1-8.

23. McMartin C, Hutchinson LE, Hyde R, Peters GE. Analysis of structural requirements for the absorption of drugs and macromolecules from the nasal cavity. $J$ Pharm Sci 1987; 76: 535-540.

24. Pires $A$, Fortuna $A$, Alves $G$, Falcão $A$. Intranasal drug delivery: How, why and what for? J Pharm Pharmaceut Sci, 2009; 12(3): 288-311.

25. Özsoy Y, Güngör S, Cevher E. Nasal delivery of high Mw drugs. Molecules, 2009; 14: 3754-3779.

26. Karmarkar AB, Gonjari ID, Hosmani $A H$, Dhabale $P N$, Thite RD. Preparation and in vitro evaluation of lyophilized nasal inserts of tramado hydrochloride.Asian J PharmSci 2008; 3(6): 27628.

27. Zhou M, Donovan M D. Intranasal mucociliary clearance of putative bioadhesive polymer gels. Int $\mathrm{J}$ Pharm 1996; 135: 115-125.

28. Suzuki Y, Makino Y. Mucosal drug delivery using cellulose derivatives as a functional polymer. $J$ Control Release 1999; 62: 101-107.

29. Vinod KR, Rohit Reddy $T$, Sandhya S, David $B$, Venkatram $R B$. Critical review on mucoadhesive drug delivery systems. Hygeia J D Med 2012; 4(1): 7-28.

30. Yadav VK, Gupta AB, Kumar R, Yadav JS, Kumar B. Mucoadhesive polymers: Means of improving the mucoadhesive properties ofdrug delivery system. $J$ Chem Pharm Res 2010; 2(5): 418-432.

31. Behl CR, Pimplaskar HK, Sileno AP, Meireles J, Romeo $V D$. Effect of physicochemical properties and other factors on systemics nasal drug delivery. Adv Drug Deliver Rev 1998; 29: 89-116.

32. Taş C, Özkan CK, Savaşer A, Özkan Y, Taşdemir U, Altunay $\mathrm{H}$. Nasal administration of metoclopramide from different dosage forms: in vitro, ex vivo, and in vivo evaluation. Drug Deliv 2009; 16(3): 167-175.

33. Vasir JK, Tambwekar K, Garg S. Bioadhesive microspheres as a controlled drug delivery system. Int J Pharm 2003; 255: 13-32.

34. Özsoy Y, Tunçel T, Can A, Akev N, Birteksöz S, Gerçeker $A$. In vivo studies on nasal preparations of ciprofloxacin hydrochloride. Pharmazie 2000; 55: 607-609.

35. Zaki NM, Awad GA, Mortada ND, Elhady SSA. Enhanced bioavailability of metoclopramide $\mathrm{HCl}$ by intranasal administration of a mucoadhesive in situ gel with modulated rheological and mucaciliary transport properties. Eur J Pharm Sci 2007; 32: 296-307.
36. Taş C, Özkan CK, Savaşer A, Özkan Y, Taşdemir $U$, Altunay $H$. Nasal absorption of metoclopramide from different Carbopol 981 based formulations: In vitro, ex vivo and in vivo evaluation. Eur $J$ Pharm Biopharm 2006; 64: 246-254.

37. Merkus FWHM, Verhoef JC. Nasal drug delivery: trends and perspectives. In: Boyian JC. [Ed.], Encyclopedia of Pharmaceutical Technology, New York, 1994, pp.191-220.

38. Bertram U, Bodmeier R. Parameters affecting the drug release from in situ gelling nasal inserts. Eur $J$ Pharm Biopharm 2006; 63: 310-319.

39. Thapa $P$, Baillie AJ, Stevens HNE. Lyophilization of unit dose pharmaceutical dosage forms. Drug Dev Ind Pharm 2003; 29(5): 595- 602.

40. Mitra $A$, Dey $B$. Chitosan microspheres in novel drug delivery systems. Indian J Pharm Sci 2011; 73(4): 355-366.

41. Mishra RK, Banthia AK, Majeed ABA. Pectin based formulations for biomedical applications: A review. Asian J Pharm Clin Res 2012; 5(4): 1-7.

42. Luppi B, Bigucci F, Cerchiara T, Zecchi V. Chitosanbased hydrogels for nasal drug delivery: from inserts to nanoparticles. Expert Opin Drug Deliv 2010; 7(7): 811-828.

43. Mclnnes F, Thapa $P$, Stevens HNE. Enhanced Nasal Delivery with Lyophilized Inserts. In: Williams RO, Taft DR, McConville JT. [Eds.], Advanced Drug Formulation Design to Optimize Therapeutic Outcomes, CRC Press, Florida, 2007, pp. 281-301,

44. Thapa $P$, Baillie AJ, Stevens HNE. Lyophilization of unit dose pharmaceutical dosage forms. Drug Dev Ind Pharm, 2003; 29: 595-602.

45. Azria M. Solid nasal drug insert. Ger Offen 1986; DE 3617728, 04 December.

46. Farid RM, Etman MA, Nada $A H$, Ebian AA. Formulation and in vitro evaluation of salbutamol sulphate in situ gelling nasal inserts. AAPS PharmSciTech, 2013; 14(2): 712-718.

47. Mclnnes FJ, Thapa PP, Baillie AJ, Welling PG, Watson DG. In vivo evulation of nicotine lyophilised nasal insert in sheep. Int J Pharm 2005; 304(1-2): 72-82.

48. Mclnnes FJ, O'Mahony B, Lindsay B, Band J, Wilson CG, Hodges LA. Nasal residence of insulin containing lyophilised nasal insert formulations, using gamma scintigraphy. Eur J Pharm Sci 2007; 31(1): 25-31.

49. Bertram U, Bodmeier R. In situ gelling, bioadhesive nasal inserts for extended drug delivery: In vitro characterization of a new nasal dosage form. Eur $J$ Pharm Sci 2006; 27: 62-71.

50. Bertram U, Bodmeier R. Effect of polymer $M w$ and of polymer blends on the properties of rapidly gelling nasal inserts. Drug Dev Ind Pharm 2012; 38(6): 659-669.

51. Karmarkar $A B$, Gonjari ID, Hosmani $A H$, Dhabale $P N$, Thite RD. Preparation and in-vitro evaluation of lyophilized nasal inserts of tramadol hydrochloride. Asian J Pharm Sci 2008; 3(6): 276-283.

52. Yenil Ö, Kahraman E, Algın Yapar E, Cevher E, Özkırımlı $S$, Özsoy Y. Preparation and evaluation of bioadhesive inserts containing verapamil hydrochloride for nasal delivery. Lat Am J Pharm 2013; 32(8): 1170-1177.

53. Werner $U$, Damgé $C$, Maincent $P$, Bodmeier $R$. Properties of in situ gelling nasal inserts containing estradiol/methyl $\beta$-cyclodextrin. J Drug Del Sci Tech 2004; 14(4): 275-284.

54. Dehghan MH, Girase M. Freeze-dried xanthan/guar gum nasal inserts for the delivery of metoclopramide hydrochloride. Iranian J Pharm Res 2012; 11(2): 513-521.

55. Bertram U, Bernard MC, Haensler J, Maincent $P$, Bodmeier $R$. In situ gelling nasal inserts for influenza vaccine delivery. Drug Dev Ind Pharm 2010; 36(5): 581-593 
56. Puapermpoonsiri U, Spencer J, van der Walle CF. A freeze-dried formulationof bacteriophage encapsulated in biodegradable microspheres. Eur J Pharm Biopharm 2009; 72: 26-33.

57. Wright A, Hawkins $\mathrm{CH}$, Anggard EE, Harper DR. A controlled clinical trial of a therapeutic bacteriophage perparation in chronic otitis due toantibiotic-resistant Pseudomonas aeruginosa; a preliminary report of efficacy. Clin Otolaryngol 2009; 34: 349-357.

58. Alfadhel M, Puapermpoonsiri U, Ford SJ, Mclnnes FJ, van der Walle CF. Lyophilized inserts for nasal administration harboring bacteriophage selective for Staphylococcus aureus: in vitro evaluation. Int $J$ Pharm, 2011; 416(1): 280- 287.

59. Luppi B, Bigucci F, Mercolini L, Musenga A, Sorrenti M. Novel mucoadhesive nasal inserts based on chitosan/hyaluronate polyelectrolyte complexes for peptide and protein delivery. The J Pharm Pharmacol 2009; 61(2): 151-157.

60. Luppi B, Bigucci F, Abruzzo A, Corace G, Cerchiara $T$, Zecchi V. Freeze-dried chitosan/pectin nasal inserts for antipsychotic drug delivery. Eur J Pharm Biopharm 2010; 75(3): 381-387.
61. Mclnnes F, Baillie AJ, Stevens HN. The use of simple dynamic mucosal models and confocal microscopy for the evaluation of lyophilised nasal formulations. J Pharm Pharmacol 2007; 59: 759-767.

62. Mercolini L, Musenga A, Saladini B, Bigucci F, Luppi B. Determination of insulin in innovative formulations by means of LC coupled to fluorescence detection. $J$ Pharm Biomedical Analysis, 2008; 48(5):13031309.

63. Bai S, Yang T, Abbruscato TJ, Ahsan F. Evaluation of human nasal RPMI 2650 cells grown at an airliquid interface as a model for nasal drug transport studies. J Pharm Sci, 2008; 97(3) : 1165-1178.

64. Agu RU, Ugwoke MI. In situ and ex vivo nasal models for preclinical drug development studies. In: Carsten E, Kwang-Jin K.(Eds.), Drug Absorption Studies, In situ, In vitro and In silico models, AAPS Press, New York, 2008, pp. 112-134.

65. Patil PR, Salve VK, Thorat RU, Puranik PK, Khadabadi SS. Modern encroachment and provocation in nasal drug delivery system. IJPSR 2013; 4(7): 2569-2575. 